\title{
Blood fatty acid changes in healthy young Americans in response to a 10-week diet that increased $n-3$ and reduced $n-6$ fatty acid consumption: a randomised controlled trial
}

\author{
Andrew J. Young ${ }^{1,2 *}$, Bernadette P. Marriott ${ }^{3,4}$, Catherine M. Champagne ${ }^{5}$, Michael R. Hawes ${ }^{6}$, \\ Scott J. Montain ${ }^{1}$, Neil M. Johannsen ${ }^{5}$, Kevin Berry ${ }^{4}$ and Joseph R. Hibbeln ${ }^{7}$ \\ ${ }^{1}$ Military Nutrition Division, US Army Research Institute of Environmental Medicine, Natick, MA O1760, USA \\ ${ }^{2}$ Oak Ridge Institute for Science and Education, Belcamp, MD 21017, USA \\ ${ }^{3}$ Department of Medicine and Psychiatry, Medical University of South Carolina, Charleston, SC 29425, USA \\ ${ }^{4}$ Samueli Institute, Alexandria, VA 22314, USA \\ ${ }^{5}$ Pennington Biomedical Research Center, Baton Rouge, LA 70808, USA \\ ${ }^{6}$ Belovo Inc., Southern Pines, NC 28387, USA \\ ${ }^{7}$ National Institutes of Health, National Institute on Alcohol Abuse and Alcoholism, Rockville, MD 20892, USA \\ (Submitted 30 December 2016 - Final revision received 27 February 2017 - Accepted 29 March 2017 - First published online 23 May 2017)
}

\section{Abstract}

Military personnel generally under-consume $n$ - 3 fatty acids and overconsume $n$ - 6 fatty acids. In a placebo-controlled, double-blinded study, we investigated whether a diet suitable for implementation in military dining facilities and civilian cafeterias could benefit $n-3 / n-6$ fatty acid status of consumers. Three volunteer groups were provided different diets for 10 weeks. Control (CON) participants consumed meals from the US Military's Standard Garrison Dining Facility Menu. Experimental, moderate (EXP-Mod) and experimental-high (EXP-High) participants consumed the same meals, but high $n-6$ fatty acid and low $n-3$ fatty acid containing chicken, egg, oils and food ingredients were replaced with products having less $n-6$ fatty acids and more $n-3$ fatty acids. The EXP-High participants also consumed smoothies containing 1000 mg $n$ - 3 fatty acids per serving, whereas other participants received placebo smoothies. Plasma and erythrocyte EPA and DHA in CON group remained unchanged throughout, whereas EPA, DHA and Omega-3 Index increased in EXP-Mod and EXP-High groups, and were higher than in CON group after 5 weeks. After 10 weeks, Omega-3 Index in EXP-High group had increased further. No participants exhibited changes in fasting plasma TAG, total cholesterol, LDL, HDL, mood or emotional reactivity. Replacing high linoleic acid (LA) containing foods in dining facility menus with similar high oleic acid/low LA and high $n-3$ fatty acid foods can improve $n-6 / n-3$ blood fatty acid status after 5 weeks. The diets were well accepted and suitable for implementation in group feeding settings like military dining facilities and civilian cafeterias.

\section{Key words: Diet interventions: Cafeterias: $\mathbf{n - 3 / n - 6}$ Fatty acids: Military personnel}

Changes in the American diet over the last century have caused marked increases in consumption of seed oils containing high amounts of $n-6$ PUFA. In particular, consumption of soyabean oil is estimated to have increased by $>1000$-fold between 1909 and 1999, tripling linoleic acid (LA) intake ${ }^{(1)}$. Consumption of seed oils and other sources of $n$-3 PUFA also increased over the twentieth century, but by much less, and $\alpha$-linolenic acid (ALA) intake is estimated to have only doubled ${ }^{(1)}$. In the body, LA competes with ALA for the enzymes that transform those PUFA into their respective highly unsaturated $n-6$ and $n-3$ fatty acid
( $n$-6 highly unsaturated fatty acid (HUFA) and n-3 HUFA) derivatives. Thus, the dramatic increase in consumption of $n-6$ PUFA could increase tissue arachidonic acid (AA) and reduce tissue EPA and DHA acids. These dietary changes have been predicted to reduce $n$ - 3 HUFA content of human tissue membranes by 27 to $54 \%{ }^{(1)}$, and observed changes in the ratio of $n-6: n-3$ PUFA content of human tissues during that period are consistent with those predictions ${ }^{(1-3)}$.

This dietary shift and accompanying tissue changes in HUFA may influence health. Metabolism of LA results in production

Disclaimer: The opinions or assertions contained herein are the private views of the authors and are not to be construed as official or as reflecting the official views of the US Army, the Department of Defense, the National Institutes of Health, the Medical University of South Carolina, The Pennington Biomedical Research Center or the Samueli Institute. Any citations or commercial organisations and trade names in this report do not constitute an official endorsement of approval of the products or services of these organisations by the above mentioned groups.

Abbreviations: AA, arachidonic acid; CON, control; EXP-High, experimental-high; EXP-Mod, experimental moderate; HUFA, highly unsaturated fatty acid; LA, linoleic acid; SCD, sudden cardiac death.

* Corresponding author: A. J. Young, fax +1 508233 5141, email andrew.j.young.ctr@mail.mil 
of AA, which in turn leads to formation of pro-inflammatory and pro-thrombotic mediators. Some research suggests that consuming high amounts of LA increases risk of cardiovascular and $\mathrm{CHD}^{(4,5)}$. Other research suggests that increases in $n-3$ fatty acid consumption may have beneficial effects on a variety of human diseases including cardiovascular ${ }^{(6,7)}$ and proinflammatory diseases ${ }^{(8,9)}$, as well as psychiatric and neurological disorders ${ }^{(10-12)}$ and possibly obesity ${ }^{(13)}$. In a postmortem, case-control study ${ }^{(14)}$, the risk of suicide among active-duty military personnel was found to increase with decreasing blood DHA levels, suggesting that this population might especially benefit from effective dietary interventions to improve their $n-3$ PUFA status. Developing new dietary approaches that enhance health, performance and resilience of service members is a research priority for the Defense Science Board of the US Department of Defense ${ }^{(15)}$, with clear translational benefits to the general population. One such approach of particular interest is to elevate levels of $n$-3, and lower $n-6$ fatty acids in military diets ${ }^{(16)}$.

Previous research has demonstrated that decreasing $n-6$ PUFA intake and increasing $n$-3 PUFA intake can improve $n$ - 3 HUFA status, but the interventions tested imposed a substantial dietary adjustment, required significant compliance monitoring and were unlikely to be feasible or effective for populations dining ad libitum ${ }^{(17-19)}$. Therefore, this study aimed to determine if a dietary adjustment suitable for implementation in group dining facilities could provide benefits for $n$ fatty acid status. We hypothesised that swapping high oleic acid/low LA foods and high $n-3$ fatty acid foods for 'like' high LA containing foods and food ingredients in the US Military's Standard 28-day Garrison Dining Facility Menu would improve blood fatty acid and lipid profiles, and indices of mood and emotional reactivity of diners. Using a modelling approach, we previously demonstrated that swapping low $n-6$ and high $n-3$ food ingredients for the standard ingredients used in that menu had the potential to increase Omega- 3 Index from 3.0 to $6.5^{(20)}$. In addition, we assessed effects of a second intervention diet employing all of the same food item swaps, but also providing a food (a 'smoothie') supplemented with $n$-3 fatty acids, to determine if additional $n$-3 fatty acid supplementation would be necessary to positively affect fatty acid and lipid profiles and mood and emotional measures. To evaluate whether the interventions would have practical application for military settings, we assessed effects of the dietary interventions after a 10-week period, to mimic the durations of many military training programs, when trainees subsist entirely or nearly entirely in a Military Garrison Dining Facility.

\section{Methods}

\section{Study design and participants}

The study was a placebo-controlled, double-blinded study in which participants were randomised into one of three parallel groups, each of which was provided a different diet to consume for 10 weeks. The participants and scientific team were blinded to randomisation groups, but study dietitians and metabolic kitchen staff were not blinded, due to the nature of the nutrition intervention. The control (CON) group consumed a daily diet in which meals were based on the standard US Military 28-day Garrison Dining Facility Menu. The experimental moderate group (EXP-Mod) consumed a daily diet comprised of the same meals, but in which high $n-6$ and low $n-3$ containing chicken, egg and oil foods and food ingredients were replaced with indistinguishable products specially produced to have lower proportion of $n-6$ and higher $n-3$ fatty acids. The experimental-high (EXP-High) group consumed the same daily diet as EXP-Mod, but also consumed a smoothie containing $1000 \mathrm{mg}$ of $n-3$ fatty acids per $200 \mathrm{ml}$ serving; the CON and EXP-Mod groups received a matching placebo smoothie. The CON, EXP-Mod and EXP-High diets all provided participants the same total energy per kg body weight and the same fractional macronutrient distribution, but as described above, differed in the quantity of $n-3$ and $n-6$ fatty acids.

In all, seventy-eight healthy male and female civilians, aged 18-40 years, living in the Baton Rouge, LA region, and not employed by the organisations sponsoring this investigation participated in this study. The Consort Diagram shown in Fig. 1 depicts the recruitment, screening, selection and randomisation of participants in the study. Participants were randomised to one of the three groups (CON, EXP-Med, EXP-High) using a computer-generated random ID number once the individual was deemed eligible for the study, signed the informed consent and health insurance portability and accountability documents, and completed screening procedures. The randomisation process balanced sex across the three study groups. Participants were all omnivores and agreed to discontinue use of dietary supplements and limit alcohol intake to moderate levels (as per Dietary Guidelines for Americans, 2010) for the duration of the study. Individuals with clinically significant disease, psychiatric disorders, medical conditions requiring use of medications, or changes in body weight $>10 \%$ over the 2 months before enrolment were excluded. Other inclusion and exclusion criteria were used to enroll a participant pool comparable in general health, age, body weight and BMI to the active-duty military population. Recruitment of participants began in August 2012, and data collection from participants was completed in November 2013.

After enrolment and assignment to study groups, participants completed baseline testing (week 0). For the next 10 weeks (feeding phase, weeks 1-10), participants consumed the prescribed CON, EXP-Mod or EXP-High diets. Baseline testing included self-reported demographics, anthropometry (body weight, height, body composition), 24-h automated multiple pass method and 30-d food frequency assessments, measurement of RMR, blood sampling for fatty acid and blood lipid analyses, and cognitive/emotional tests. Race/ethnicity was self-reported by the participants using the multiple question approach recommended by the US Office of Management and Budget for human research in the $\mathrm{USA}^{(21)}$. Blood sampling for fatty acid and blood lipid analyses, and cognitive/emotional tests were repeated during the feeding phase in weeks 5 and again in week 10, and each participant's body weight was recorded daily, except for weekends during feeding phase of the study. Body composition was reassessed in week 10 .

This study was conducted at the Pennington Biomedical Research Center in Baton Rouge, LA, according to the guidelines laid down in the Declaration of Helsinki, and all procedures 
involving human participants were reviewed and approved by the Pennington Human Subjects Institutional Review Board. All volunteers provided signed, informed consent to participate in the study. All foods consumed by participants during the feeding phase of the study were prepared in the metabolic kitchen of the Pennington Biomedical Research Center. This trial was registered at ClinicalTrials.gov PRS with the identifier NCT01642368.

\section{Dietary interventions}

The standard US Military 28-day Garrison Dining Facility Menu ${ }^{(22)}$ was used to create an 8-d menu cycle, with twenty-four meals, each of which could be configured to provide the same nutrient distribution at four levels of energy content (6276, 8368, 10460 , $12552 \mathrm{~kJ}(1500,2000,2500$ and $3000 \mathrm{kcal}))$. Individual food items were prepared according to the US Armed Forces Recipe Services Index ${ }^{(23)}$. As described above, the foods consumed by the CON, EXP-Mod and EXP-High diet groups were indistinguishable and followed the same 8-d menu plan. However, the EXP-Mod and EXP-High diets substituted commercially produced high oleic acid/low LA foods and foods containing high amounts of $n-3$ HUFA for 'like' foods in the standard CON diet that contained high amounts of LA and low amounts of $n-3$ HUFA. The low $n-6 /$ high $n-3$ fatty acid containing foods and food items substituted into the intervention diets included eggs, chicken, albacore tuna, grass-fed beef, peanut butter, cooking oils and condiments (dressings, spreads and sauces). In addition, the EXP-High group was provided high $n$ - 3 HUFA containing smoothie drinks, and the other two groups were provided matching placebo smoothie drinks. The specialty food products were commercially available or produced specifically for this study (chicken and eggs), and provided for use in the study at no cost by a number of vendors, whose contributions are acknowledged at the end of this paper. All of the food products were produced, stored and shipped to Pennington Biomedical Research Center according to US Department of Agriculture standards. The online Supplementary Tables S1 and S2 provide a detailed listing of the fatty acid composition of the individual foods provided to each of the participant groups during the course of the study.

The diets were individually adjusted for each participant to ensure that total daily energy intake was sufficient to maintain constant body weight over the 10 -week study. Individual daily energy requirements were initially estimated as the average of the measured RMR and the predicted RMR using the Mifflin equation $^{(24)}$, both multiplied by an activity factor of 1.5 , and then rounded to the nearest $418 \mathrm{~kJ}(100 \mathrm{kcal})$. If the participant's energy requirement fell between two of the menu's standardised energy content levels, unit foods matching the overall macronutrient distribution (including fatty acids) of the menu were added to the lower energy content menu in $418 \mathrm{~kJ}$ (100 kcal) increments until the desired energy intake was achieved. For the duration of the 10-week study, participants consumed weekday breakfast and dinner meals onsite at Pennington Biomedical Research Center, and were provided with lunch and weekend meals packed 'to go'. Participants were required to consume all foods provided at each meal, and compliance was monitored by Pennington's dietetic support staff who supervised consumption of meals onsite. For meals provided for offsite consumption participants completed a checklist of items consumed at each meal, and any additional items consumed that were not provided by study staff, and when they returned to the Center for their next onsite meal, they provided this checklist to the dietetic staff for review and monitoring of dietary compliance. Each participant's body weight was measured each weekday morning when he/she arrived for breakfast, and individual diets were adjusted as needed throughout the study to increase or decrease energy content if body weight appeared to be increasing or decreasing.

Each individual's daily energy and macronutrient intakes were estimated from foods consumed using computerised dietary nutrient analysis software (the Pennington MENu database, an inhouse database and software system that uses the US Department of Agriculture's National Nutrient Database for Standard Reference). In addition, the fatty acid composition of each food and the average fatty acid composition of the volunteers' daily diet were determined. At the Pennington Metabolic kitchen, each type of food used in the diets and a homogenate of all foods comprising each of the $8 \mathrm{~d}$ of the menu cycle were frozen at $-40^{\circ} \mathrm{C}$ and shipped to the National Institute on Alcoholism and Alcohol Abuse, National Institutes of Health for analysis. Three samples of each type of food and each homogenate were thawed, weighed on an analytic balance and immediately placed into $2 \mathrm{ml}$ cold methanol that contained $50 \mathrm{mg}$ butylated hydroxytoluene $/ \mathrm{ml}$. A known mass of $22: 3 n-3$ methyl ester internal standard (Nu-Chek-Prep) was added to the solution. These samples in solution were homogenised mechanically with the TH-01 handheld homogenizer (OMNI International), chilled in an outer ice bath, and subsequently capped under N. Fatty acids were extracted from these food and homogenate samples using a modification of the method of Folch et al. $^{(25)}$ by using chloroform-methanol solvents in a 1:1 ratio and an aqueous phosphate buffer solution. Lipids were recovered into the chloroform layer after two extractions. This fraction was evaporated under $\mathrm{N}$ and transesterified ${ }^{(26)}$ The fatty acid analyses employed high-throughput robotic direct methylation coupled with fast GLC using an Agilent Technologies 7890 with a flame ionization detector ${ }^{(27)}$. Peaks were identified by using authentic standards (Nu-Check-Prep). Fatty acids were quantified by comparison with peak areas of the identified $22: 3 n-3$ internal standard peaks. The analysis has an inter-assay variance of $<0.5 \%$ fatty acid composition. The average daily fatty acid intake was obtained by averaging the fatty acid content of the foods provided for each of the $8 \mathrm{~d}$, and then multiplying by the average amount of food consumed each day during the dietary intervention period.

After completing each week of the study, the participants were asked to rate their satisfaction with their diet. The participants' diet satisfaction ratings were obtained using a numerical scale. The scale ranged from 1 to 7 , and was anchored with the written cues 'dislike extremely', 'neither like nor dislike', and 'like extremely' at the values of 1,4 and 7 , respectively.

\section{Blood sampling and analyses}

To evaluate the effect of the dietary intervention on blood lipids and fatty acids, blood samples were obtained from participants during the baseline testing (week 0), midway through the study 
(week 5) and at the end of the study (week 10). Blood samples for determination of fatty acid concentrations in plasma and erythrocytes and for plasma total TAG, cholesterol, HDL and LDL concentrations were obtained by venepuncture with the participants in the seated position following a 12-h fast. Samples were collected into a $10 \mathrm{ml}$ draw tube containing K2EDTA. All blood samples were immediately placed on ice and transported to the laboratory for specimen processing. The draw tubes were centrifuged at $4^{\circ} \mathrm{C}, 2095$ relative centrifugal force $(\boldsymbol{g})$ for $10 \mathrm{~min}$ in a Beckman Coulter X-15R centrifuge with swinging buckets. Aliquots $(0.5 \mathrm{ml})$ of plasma and erythrocytes were transferred to polypropylene micro tubes utilising caps with o-ring closure, and placed in frozen storage within $1 \mathrm{~h}$ of sample collection and stored at $-70^{\circ} \mathrm{C}$, in a Thermo Fisher Scientific ultra-low freezer model 907.

Samples for plasma and erythrocyte fatty acid analysis were shipped frozen on dry ice to the Section of Nutritional Neurosciences, Laboratory of Membrane Biochemistry and Biophysics, National Institute on Alcoholism and Alcohol Abuse, National Institutes of Health for fatty acid analysis. Plasma and erythrocyte samples were assayed for fatty acid composition in total lipids as previously described ${ }^{(27)}$. The values of fatty acids in total lipids of plasma and erythrocytes are presented as concentration $(\mathrm{nmol} / \mathrm{ml})$. The Omega-3 Index was calculated as the sum of the erythrocyte EPA and DHA content expressed as a percentage of the total erythrocyte fatty acid content ${ }^{(28)}$.

Samples for plasma total TAG, cholesterol, HDL and LDL concentrations were shipped frozen on dry ice to the US Army Research Institute of Environmental Medicine. Lipid profiles were assayed on a Siemens Dimension Xpand Plus Integrated Chemistry System, using Siemens automated flex cartridges for TAG, cholesterol, HDL and LDL (Siemens Healthcare Diagnostics). Each sample was run in duplicate. The inter-assay CV were $4.94,1.74,3.88$ and $5.04 \%$ for TAG, cholesterol, HDL and LDL, respectively.

\section{Anthropometric measurements}

Body weights were measured to the nearest $0 \cdot 1 \mathrm{~kg}$ on a GSE 460 scale. Participants were instructed to remove all outer clothing (i.e. jackets, sweaters and any heavy items carried in pockets change, phones, wallets, etc.) and shoes before weight measurement. Two body weight measurements were recorded, and averaged for use in the BMI calculation. Height was measured to the nearest $0 \cdot 1 \mathrm{~cm}$ using a wall-mounted stadiometer (Holtain Limited). Heights were measured without shoes (in socks or bare feet) with heels, buttocks and upper part of back in contact with the stadiometer. The participant was instructed to take a breath and hold while remaining flat footed. Two height measurements were recorded, with the average used for the BMI calculation (weight $(\mathrm{kg}) /$ square of height $\left(\mathrm{m}^{2}\right)$ ). Body composition was estimated using dual-energy X-ray absorptiometry (Lunar iDXA; General Electric), which provided an estimation of each participant's lean and fat mass.

\section{Mood/emotional state}

To evaluate the effect of the dietary intervention on mood and emotional state, participants completed a series of standardised tests during the baseline testing (week 0), midway through the study (week 5) and at the end of the study (week 10). The mood/emotional tests completed included the Profile of Mood States (POMS), the State-Trait Anxiety Inventory (STAI) and the Beck Depression Inventory, Second Edition (BDI-II).

The POMS is a widely used, standardised inventory of subjective mood state ${ }^{(29)}$. The POMS asks respondents to use a five-point Likert scale to describe the extent to which their current mood state ('How are you feeling right now?') corresponds to a series of sixty-five mood-related adjectives. The adjectives are grouped into six mood subscales (tension, depression, anger, vigour, fatigue and confusion ${ }^{(30)}$.

The STAI measures the severity of the overall anxiety level. The survey is divided into two subsections, each having twenty questions, to which a four-point Likert scale is used by respondents to assess two types of anxiety. The first subsection measures state anxiety (STAI-S), and the second subsection measures trait anxiety (STAI-T). The range of scores on each scale is 20-80, the higher the score indicating greater anxiety $^{(31)}$. Standards exist for different populations. This scale allows comparison of mean scores in groups of subjects, or to distinguish groups with high anxiety by choosing a cut-off. STAI-S is typically used to measure individual anxiety in response to external situation for comparison with an individual's overall STAI-T, which is relatively stable.

The BDI-II is a twenty-one-item, self-report instrument that has been used for 35 years to identify and assess depressive symptoms, and has been reported to be highly reliable regardless of the population ${ }^{(32)}$. The respondent is asked to consider each statement as it relates to the way they have felt for the past 2 weeks. Each of the twenty-one items corresponds to a symptom of depression, and is summed to give a single score for the BDI-II. The BDI-II is used to identify the presence and severity of symptoms consistent with the depression criteria of the Diagnostic and Statistical Manual of Mental Disorders, 4 th edition. There is a four-point scale for each item ranging from 0 to 3 . Total score of $0-13$ is considered minimal range, 14-19 is mild, 20-28 is moderate and 29-63 is severe.

In addition to the mood and emotional reactivity tests described above, participants completed a number of cognitive tests during baseline testing, and during the 5th and 10th week of the study. However, due to a technical error, the data from those tests were lost and will not be reported. The cognitive tests performed were: the revised eye test ${ }^{(33)}$, the emotion hexagon test ${ }^{(34)}$, the facial emotion recognition $\operatorname{task}^{(35)}$, the emotional stroop test ${ }^{(36)}$, the emotional face stroop test ${ }^{(37)}$, the immediate memory task/delayed memory task ${ }^{(38)}$.

\section{Statistical methods}

The primary outcome of interest in this study was whether the 10-week dietary intervention would significantly reduce erythrocyte $n$ - 6 HUFA concentrations and increase $n$ - 3 HUFA concentrations. We assumed that initially (i.e. pre-intervention), the percentage of total HUFA comprised of $n-6$ HUFA would average $77 \%$, with the standard deviation conservatively estimated as $6 \%^{(1)}$, and that participants in CON group would exhibit no change in that metric during the intervention. 
Based on modelling ${ }^{(20)}$, the percentage of total plasma HUFA comprised of $n-6$ HUFA was predicted to average 57 and $32 \%$ for participants in the EXP-Mod and EXP-High groups, respectively after the 10 -week intervention. We estimated (GPower 3.1.2) that the sample size required to have $90 \%$ power for detecting these differences among groups using multiple comparisons ( $t$ tests, two independent samples, Bonferroni adjustments) was ten per group. However, to ensure adequate power for detecting smaller differences (i.e. $\pm 1 \mathrm{SD}$ ) in that metric, as well as differences in the more variable measures associated with secondary outcomes (i.e. anthropometrics, mood, emotion and cognitive state, blood lipids) and adjunct studies (exercise tolerance and satiety), the sample size was increased to twenty-two per group, with a further adjustment to twenty-five to account for an expected dropout rate of $10 \%$.

Statistical analysis of data from participants who completed the study was performed using commercially available statistical software (IBM SPSS version 21). Descriptive statistics are presented as mean values and standard deviations except where specified. A mixed model ANOVA split-plot analysis was used to examine differences between diet groups over the 10-week dietary intervention, with diet group as the between subject factor and time as the within-subject factor. Identified differences between groups, time or interaction were examined post hoc using least squares differences test. A one-way ANOVA was used to examine differences in diet composition between groups as well as group comparisons made only at baseline. In the event that analysis didn't comply with the assumption of sphericity, Greenhouse-Geiser or Huynh-Feldt adjustments were utilised for $P$ value. For all analyses, a $P<0.05$ was used for establishing significant differences.

\section{Results}

\section{Baseline characteristics of participants}

As shown in Fig. 1, seventy-one participants completed the study out of the seventy-eight initially enrolled. Five participants withdrew (one from EXP-Mod, four from EXP-High) citing a variety of personal reasons not specifically related to the study procedures (e.g. schedule conflicts, transportation problems). Two participants (one from each of the EXP-High and EXP-Mod) withdrew $2 \mathrm{~d}$ after beginning the intervention diet citing gastrointestinal symptoms which they attributed to the foods being provided. Table 1 shows the sex, racial/ethnicity, age and anthropometric characteristics of the completing participants. There were no significant differences among groups for any of these characteristics. In addition, body weight, body composition and BMI all remained unchanged from the baseline values at the end of the study (week 10).

\section{Energy and nutrient intake}

Table 2 shows the total daily energy, carbohydrate, protein, fat and fatty acids provided to participants by the CON, EXP-Mod and EXP-High diets. In order to maintain stable body weight throughout the study, average daily energy provided to participants consuming the EXP-High diet was slightly (approximately $209 \mathrm{~kJ}(50 \mathrm{kcal})$ ) but significantly higher than that provided to participants consuming the EXP-Mod diet. The macronutrient

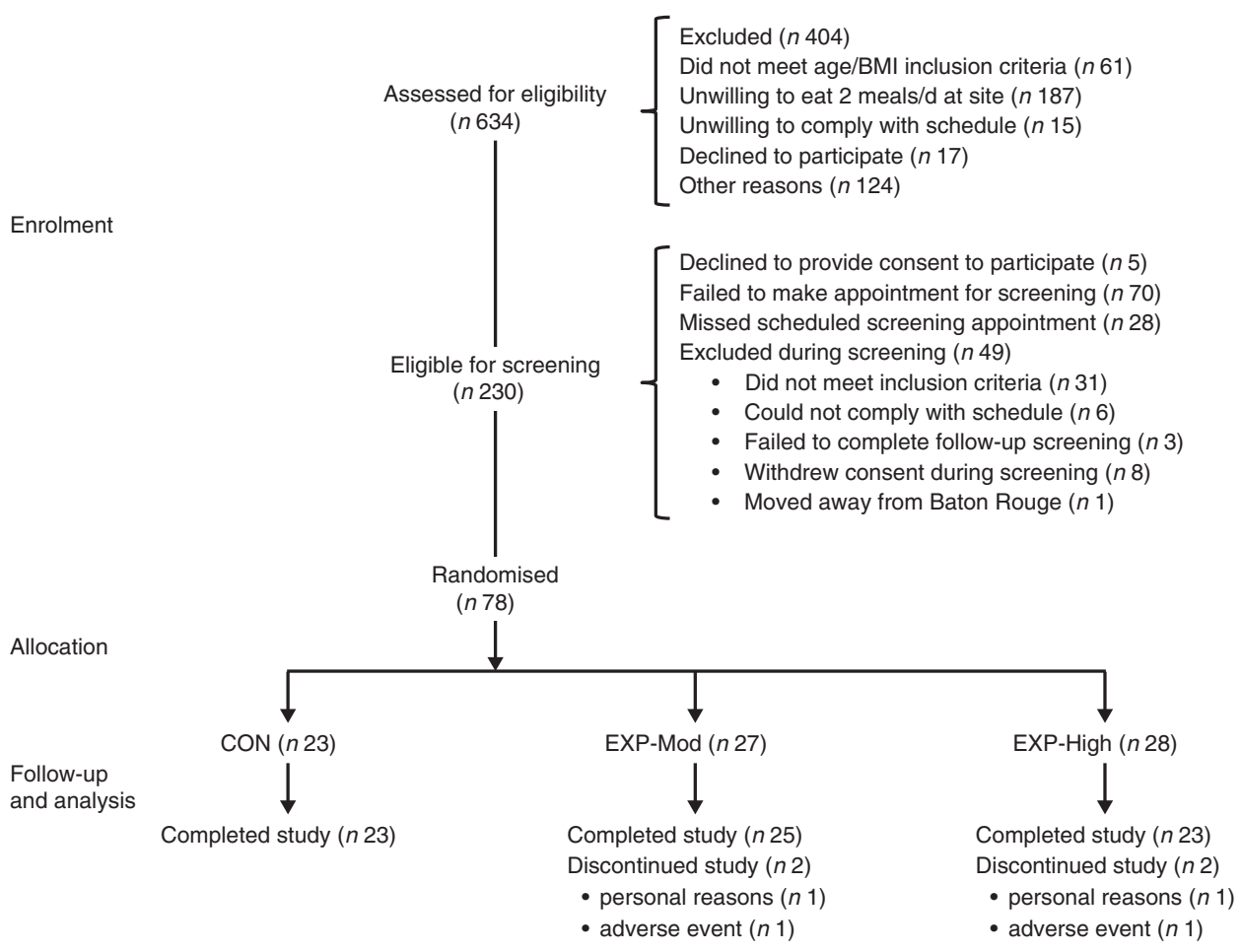

Fig. 1. Screening, enrolment, selection and randomisation of participants into control (CON), experimental, moderate $n-3$ supplemented group (EXP-Mod) and experimental-high $n-3$ supplemented group (EXP-High). 
Table 1. Characteristics of participants completing the study (Percentages and numbers; mean values and standard deviations)

\begin{tabular}{|c|c|c|c|c|c|c|c|c|}
\hline \multirow[b]{3}{*}{ Characteristics } & & & \multicolumn{6}{|c|}{ Participant groups } \\
\hline & \multicolumn{2}{|c|}{ Total sample $(n 71)$} & \multicolumn{2}{|c|}{$\mathrm{CON}$} & \multicolumn{2}{|c|}{ EXP-Mod } & \multicolumn{2}{|c|}{ EXP-High } \\
\hline & $\%$ & $n$ & $\%$ & $n$ & $\%$ & $n$ & $\%$ & $n$ \\
\hline \multicolumn{9}{|l|}{ Sex } \\
\hline Male & 52 & 37 & 52 & 12 & 52 & 13 & 52 & 12 \\
\hline Female & 48 & 34 & 48 & 11 & 48 & 12 & 48 & 11 \\
\hline \multicolumn{9}{|l|}{ Race/ethnicity } \\
\hline Asian & 10 & 7 & 0 & 0 & 16 & 4 & 13 & 3 \\
\hline Black or African-American & 14 & 10 & 22 & 5 & 12 & 3 & 9 & 2 \\
\hline Other & 14 & 3 & 13 & 3 & 0 & 0 & 0 & 0 \\
\hline \multirow[t]{2}{*}{ White } & 72 & 51 & 65 & 15 & 72 & 18 & 78 & 18 \\
\hline & Mean & SD & Mean & SD & Mean & SD & Mean & SD \\
\hline Age (years) & $26 \cdot 6$ & 4.9 & $28 \cdot 3$ & 5.5 & $27 \cdot 3$ & 5.6 & $26 \cdot 6$ & 4.9 \\
\hline \multicolumn{9}{|l|}{ Anthropometrics } \\
\hline Weight (kg) & $69 \cdot 2$ & $13 \cdot 3$ & 71.8 & $10 \cdot 1$ & $72 \cdot 0$ & 14.4 & $69 \cdot 7$ & 13.4 \\
\hline Height $(\mathrm{cm})$ & $170 \cdot 7$ & 11.0 & $170 \cdot 3$ & $9 \cdot 8$ & $174 \cdot 2$ & 9.5 & $170 \cdot 7$ & $11 \cdot 0$ \\
\hline $\mathrm{BMI}^{*}$ & 23.7 & 2.5 & 24.7 & 2.6 & 23.5 & 3.0 & 23.7 & 2.5 \\
\hline
\end{tabular}

CON, control; EXP-Mod, experimental, moderate $n-3$ supplemented group; EXP-High, experimental, high $n-3$ supplemented group. ${ }^{*} \mathrm{BMI}=$ weight $(\mathrm{kg}) /$ height $^{2}\left(\mathrm{~m}^{2}\right)$.

Table 2. Daily energy and nutrient intake* (Mean values and standard deviations)

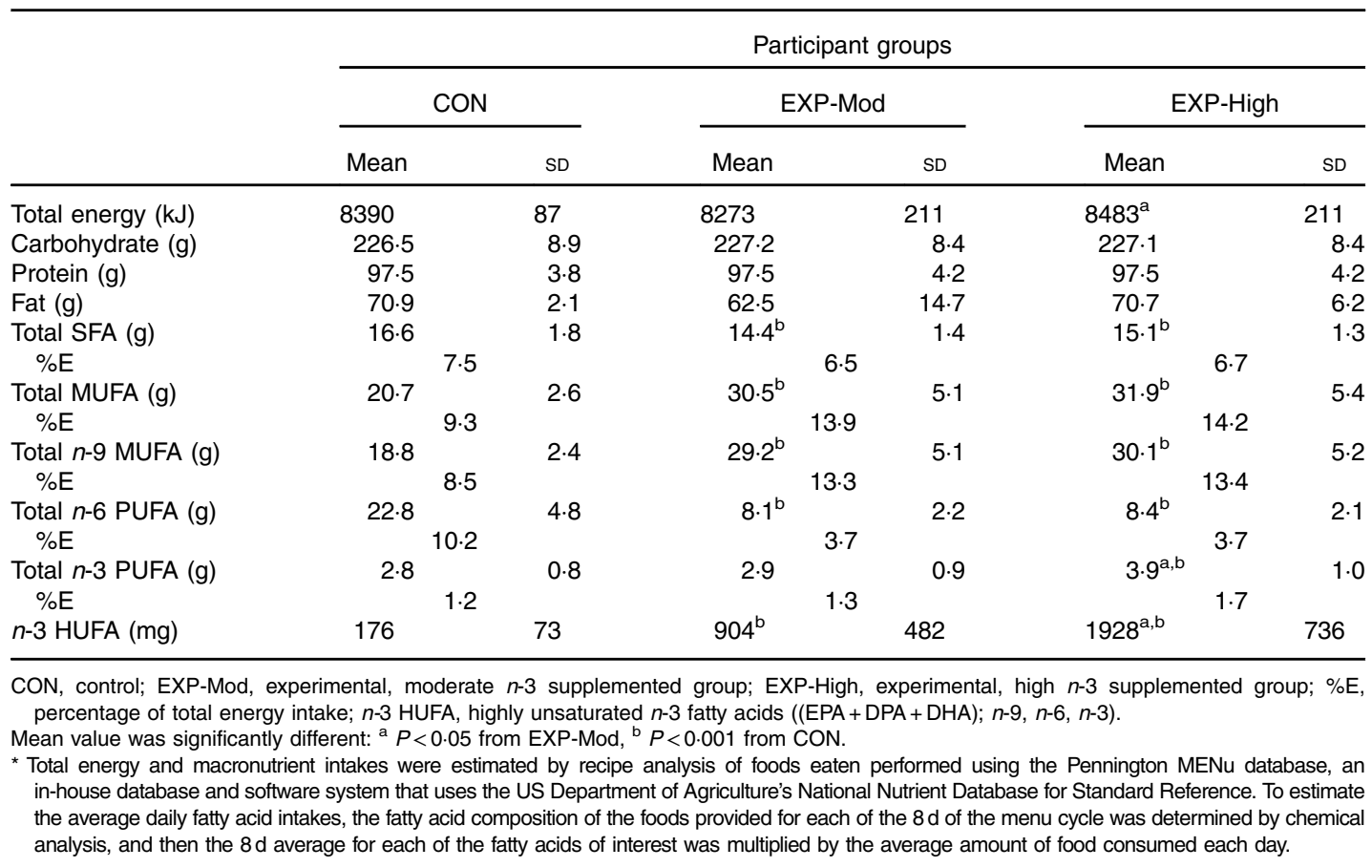

content of the three diets did not differ. As shown in Table 2, there were significant differences in the fatty acid content of the three diets. The EXP-Mod and EXP-High diets were lower in SFA, and higher in MUFA, particularly $n-9$ (primarily oleic acid), than the CON diet. Both the EXP-Mod and EXP-High diets were considerably lower in $n-6$ PUFA than the CON diet. In particular, LA intake averaged 3.3 and $3.2 \%$ energy intake for participants in the EXP-Mod and EXP-High groups, respectively, compared with $8.7 \%$ of energy intake in the CON group participants. The $n-3$
PUFA content of the EXP-High diet was higher than both the CON and EXP-Mod diets, which did not differ. The $n$ - 3 HUFA content of the EXP-Mod and EXP-High diets was higher than that of the CON diet, and the $n-3$ HUFA content of the EXP-High diet was higher than that of the EXP-Mod diet.

There were no differences between groups in the participants' ratings of satisfaction with their prescribed diet at the end of each week of the study. Overall, satisfaction ratings averaged 5.02 (SD 1.04) after the 1st week on the diets, but after the 4th week 
on the diet, satisfaction ratings had declined somewhat to 4.72 (SD 1.14), and ratings remained lower for the remainder of the study compared with after the 1st week. Despite the slight decline in satisfaction ratings over the course of the study, the ratings remained above 4 , the neutral point on the ratings scale.

\section{Blood fatty acids}

Table 3 shows the plasma fatty acid concentrations $(\mathrm{nmol} / \mathrm{ml})$ for each of the participant groups before and after 5 and 10 weeks of the dietary intervention. Plasma SFA did not change as a result of the dietary intervention, and there were no differences in plasma SFA between groups at any time. There were no differences in MUFA between groups before the dietary intervention began, but changes in MUFA during the intervention differed between groups. CON exhibited a decrease in plasma MUFA, and MUFA concentrations were less than in EXP-Mod and EXP-High. The decrease in MUFA in CON was largely attributable to a decrease in oleic acid, between weeks 0 and 5, which persisted through week 10. In contrast, MUFA of the EXP-Mod and EXPHigh remained unchanged (except for nervonic acid, which increased) throughout the dietary intervention. Plasma $n-6$ PUFA did not differ between groups before the dietary intervention. However, after 5 weeks of the intervention, total plasma $n-6$ PUFA in the CON remained unchanged (except for adrenic acid which decreased slightly), whereas in the EXP-Mod and EXPHigh groups, plasma $n-6$ PUFA decreased and were lower than in CON, principally due to decreased linoleic and AA, and those changes persisted through week 10. Plasma $n$-3 PUFA did not differ between groups before the dietary intervention, and remained unchanged throughout the intervention in the CON group. However, in both the EXP-Mod and EXP-High groups, plasma $n$-3 PUFA increased after 5 weeks of the intervention, and the increase persisted through week 10 of the intervention. The increased $n$-3 PUFA were primarily attributable to large increases in the $n$-3 HUFA, EPA and DHA, although DPA was also increased in the EXP-High group. Further, during both week 5 and week 10 of the intervention, plasma n-3 HUFA were higher in the EXP-High than EXP-Mod group After week 10 of the intervention, the percentage of total HUFA comprised of $n-6$ HUFA (not shown in table) in the CON group averaged 82 (SD 1)\%, which was not different from week 0 before the intervention. In contrast, for the EXP-Mod and EXP-High groups, the percentage of total HUFA comprised of $n$ - 6 HUFA after week 10 of the intervention averaged 64 (sD 6) and 54 (sD 5)\% $(P<0.05)$, respectively, both of which were lower $(P<0.05)$ than their respective week 0 values and lower $(P<0.05)$ than in CON at that same time.

Changes in erythrocyte fatty acid concentrations of the CON, EXP-Mod and EXP-High groups during the 10-week

Table 3. Plasma fatty acid concentrations $(\mathrm{nmol} / \mathrm{ml})$

(Mean values and standard deviations)

\begin{tabular}{|c|c|c|c|c|c|c|c|c|c|c|c|c|c|c|c|c|c|c|}
\hline \multirow[b]{3}{*}{ Fatty acids } & \multicolumn{6}{|c|}{ Week 0} & \multicolumn{6}{|c|}{ Week 5} & \multicolumn{6}{|c|}{ Week 10} \\
\hline & \multicolumn{2}{|c|}{ CON } & \multicolumn{2}{|c|}{ EXP-Mod } & \multicolumn{2}{|c|}{ EXP-High } & \multicolumn{2}{|c|}{ CON } & \multicolumn{2}{|c|}{ EXP-Mod } & \multicolumn{2}{|c|}{ EXP-High } & \multicolumn{2}{|c|}{ CON } & \multicolumn{2}{|c|}{ EXP-Mod } & \multicolumn{2}{|c|}{ EXP-High } \\
\hline & Mean & SD & Mean & SD & Mean & SD & Mean & SD & Mean & SD & Mean & SD & Mean & SD & Mean & SD & Mean & SD \\
\hline \multicolumn{19}{|l|}{ SFA } \\
\hline Palmitic $^{*}(16: 0)$ & 1998 & 436 & 2058 & 346 & 2068 & 474 & 1918 & 418 & 1921 & 364 & 1890 & 496 & 1897 & 371 & 1919 & 415 & 2013 & 579 \\
\hline Stearic $(18: 0)$ & 645 & 73 & 683 & 95 & 683 & 127 & 666 & 91 & 662 & 86 & 647 & 86 & 662 & 73 & 676 & 115 & 685 & 132 \\
\hline Behenic† $(22: 0)$ & 67 & 12 & 62 & 13 & 64 & 12 & 73 & 11 & 65 & 13 & 63 & 12 & 72 & 9 & 64 & 15 & 63 & 12 \\
\hline Lignoceratic (24:0) & 55 & 11 & 53 & 10 & 57 & 11 & 57 & 10 & 55 & 11 & 55 & 11 & 58 & 8 & 54 & 12 & 55 & 1 \\
\hline \multicolumn{19}{|l|}{ MUFA } \\
\hline Palmitoleic* $(16: 1, n-7)$ & 110 & 52 & 133 & 70 & 131 & 82 & 85 & 32 & 97 & 38 & 106 & 81 & 82 & 29 & 103 & 39 & 129 & 123 \\
\hline Oleic (18: & 1441 & 347 & 1466 & 429 & 1393 & 335 & $1118^{a}$ & 259 & $1481^{\mathrm{b}}$ & 298 & $1421^{\mathrm{b}}$ & 433 & $1097^{\mathrm{a}}$ & 232 & $1593^{b}$ & 463 & $1529^{b}$ & 458 \\
\hline ic $(18: 1, n-7)$ & 127 & 40 & 136 & 30 & 135 & 29 & 136 & 30 & $120^{a, b}$ & 22 & $117^{\mathrm{a}, \mathrm{b}}$ & 27 & 128 & 26 & 125 & 33 & $120^{\mathrm{a}}$ & 28 \\
\hline Gondoic $(20: 1, n-9)$ & 12 & 4 & 12 & 4 & 12 & 4 & 10 & 3 & 12 & 4 & 13 & 4 & 10 & 3 & 12 & 4 & 14 & \\
\hline Erucic $\ddagger(22: 1, n-9)$ & 4 & 1 & 6 & 5 & 4 & 2 & 6 & 3 & 7 & 4 & 6 & 4 & 5 & 1 & 6 & 3 & 5 & \\
\hline Nervonic $(24: 1, n-9)$ & 70 & 9 & 74 & 13 & 75 & 17 & $63^{\mathrm{a}}$ & 12 & $81^{a, b}$ & 16 & $86^{a, b}$ & 17 & $62^{\mathrm{a}}$ & 9 & $80^{a, b}$ & 16 & $90^{\mathrm{a}, \mathrm{b}, \mathrm{c}}$ & 20 \\
\hline \multicolumn{19}{|l|}{$n-6$ PUFA } \\
\hline LA $(18: 2, n-6)$ & 2640 & 597 & 2609 & 363 & 2764 & 673 & 2852 & 551 & $2313^{a, b}$ & 386 & $2249^{a, b}$ & 528 & 2840 & 466 & $2488^{a, b}$ & 548 & $2296^{\mathrm{a}, \mathrm{b}}$ & 50 \\
\hline Eicosadinoic $(20: 2, n-6)$ & 20 & 7 & 19 & 5 & 21 & 8 & 31 & 23 & 23 & 17 & 24 & 15 & 26 & 12 & 23 & 18 & 28 & \\
\hline $\operatorname{DGLA}^{*}(20: 3, n-6)$ & 116 & 39 & 105 & 30 & 114 & 31 & 90 & 33 & 85 & 32 & 84 & 35 & 95 & 34 & 93 & 34 & 87 & 31 \\
\hline AA $(20: 4, n-6)$ & 629 & 150 & 584 & 132 & 625 & 152 & 672 & 210 & $499^{a, b}$ & 92 & $495^{a, b}$ & 104 & 671 & 214 & $486^{a, b}$ & 123 & $471^{a, b}$ & 98 \\
\hline Adrenic $(22: 4, n-6)$ & 19 & 5 & 17 & 5 & 19 & 5 & $16^{\mathrm{a}}$ & 4 & $9^{a, b}$ & 2 & $8^{\mathrm{a}, \mathrm{b}}$ & 2 & $17^{\mathrm{a}}$ & 5 & $9^{a, b}$ & 3 & $8^{a, b}$ & \\
\hline Osbond $(22: 5$ & 27 & 15 & 23 & 7 & 25 & 10 & 28 & 7 & 15 & 5 & 12 & 5 & 26 & 7 & 13 & 5 & 15 & \\
\hline \multicolumn{19}{|l|}{$n-3$ PUFA } \\
\hline ALA $\ddagger(18: 3, n-3)$ & 47 & 21 & 46 & 14 & 49 & 23 & 58 & 21 & 58 & 16 & 53 & 22 & 59 & 16 & 68 & 32 & 56 & 19 \\
\hline EPA $(20: 5, n-3)$ & 28 & 14 & 30 & 16 & 28 & 14 & 21 & 7 & $87^{\mathrm{a}, \mathrm{b}}$ & 31 & $189^{a, b, c}$ & 60 & 25 & 10 & $90^{a, b}$ & 36 & $191^{\mathrm{a}, \mathrm{b}, \mathrm{c}}$ & 67 \\
\hline DPA $(22: 5, n-3)$ & 30 & 9 & 31 & 9 & 30 & 11 & 24 & 7 & 28 & 7 & $38^{a, b, c}$ & 13 & 25 & 7 & 30 & 9 & $40^{a, b, c}$ & 12 \\
\hline DHA $(22: 6, n-3)$ & 99 & 26 & 97 & 34 & 100 & 36 & 117 & 33 & $217^{a, b}$ & 57 & $275^{\mathrm{a}, \mathrm{b}, \mathrm{c}}$ & 78 & 115 & 31 & $225^{a, b}$ & 74 & $280^{a, b, c}$ & 75 \\
\hline
\end{tabular}

CON, control; EXP-Mod, experimental, moderate $n$-3 supplemented group; EXP-High, experimental, high $n$-3 supplemented group; LA, linoleic acid; DGLA, dihomo- $\gamma$-linolenic acid; AA, arachidonic acid; ALA, $a$-linolenic acid.

Mean value was significantly different: ${ }^{\mathrm{a}} P<0.05$ from corresponding group average for week $0,{ }^{\mathrm{b}} P<0.05$ from average values for CON at the same time point, ${ }^{\mathrm{c}} P<0.05$ from average values for EXP-Mod at the same time point.

* Main effect of time for palmitic, palmitoleic and DGLA, week $0>$ weeks 5 and 10.

† Main effect of group for behenic acid, CON $>$ EXP-Mod, EXP-High.

¥ Main effect of time for erucic acid and ALA, week $0<$ week 5 and week 10 . 
Table 4. Erythrocyte fatty acid concentrations (nmol/ml)

(Mean values and standard deviations)

\begin{tabular}{|c|c|c|c|c|c|c|c|c|c|c|c|c|c|c|c|c|c|c|}
\hline \multirow[b]{3}{*}{ Fatty acids } & \multicolumn{6}{|c|}{ Week 0} & \multicolumn{6}{|c|}{ Week 5} & \multicolumn{6}{|c|}{ Week 10} \\
\hline & \multicolumn{2}{|c|}{$\mathrm{CON}$} & \multicolumn{2}{|c|}{ EXP-Mod } & \multicolumn{2}{|c|}{ EXP-High } & \multicolumn{2}{|c|}{$\mathrm{CON}$} & \multicolumn{2}{|c|}{ EXP-Mod } & \multicolumn{2}{|c|}{ EXP-High } & \multicolumn{2}{|c|}{$\mathrm{CON}$} & \multicolumn{2}{|c|}{ EXP-Mod } & \multicolumn{2}{|c|}{ EXP-High } \\
\hline & Mean & SD & Mean & SD & Mean & SD & Mean & SD & Mean & SD & Mean & SD & Mean & SD & Mean & SD & Mean & SD \\
\hline \multicolumn{19}{|l|}{ SFA } \\
\hline Palmitic* $(16: 0)$ & 1536 & 113 & 1508 & 131 & 1517 & 133 & 1499 & 131 & 1481 & 134 & 1458 & 80 & 1507 & 134 & 1472 & 129 & 1436 & 130 \\
\hline Stearic $(18: 0)$ & 603 & 102 & 587 & 62 & 589 & 59 & 617 & 82 & 590 & 68 & 593 & 71 & 621 & 91 & 583 & 63 & 559 & 70 \\
\hline Behenict $(22: 0)$ & 97 & 12 & 93 & 13 & 92 & 12 & 99 & 14 & 93 & 10 & 92 & 12 & 99 & 9 & 89 & 11 & 85 & 11 \\
\hline Lignoceratict $(24: 0)$ & 258 & 39 & 254 & 27 & 247 & 30 & 258 & 28 & 255 & 25 & 248 & 29 & 270 & 33 & 254 & 33 & 236 & 25 \\
\hline \multicolumn{19}{|l|}{ MUFA } \\
\hline Palmitoleic acid $(16: 1, n-7)$ & 22 & 5 & 23 & 7 & 22 & 7 & 19 & 5 & 20 & 5 & 19 & 5 & 20 & 4 & 20 & 5 & 20 & 10 \\
\hline Oleic $(18: 1, n-9)$ & 691 & 65 & 690 & 58 & 666 & 149 & $619^{a}$ & 48 & $742^{a, b}$ & 88 & $707^{b}$ & 62 & $614^{a}$ & 59 & $745^{a, b}$ & 74 & $703^{b}$ & 78 \\
\hline Vaccenic $(18: 1, n-7)$ & 68 & 9 & 66 & 7 & 66 & 7 & 72 & 8 & $64^{a, b}$ & 7 & $63^{a, b}$ & 6 & 70 & 6 & $61^{a, b}$ & 5 & $60^{\mathrm{a}, \mathrm{b}}$ & 7 \\
\hline Gondoic $(20: 1, n-9)$ & 11 & 1 & 10 & 2 & 11 & 4 & 11 & 2 & 12 & 4 & 12 & 3 & 10 & 1 & 11 & 2 & 12 & 2 \\
\hline Erucic $(22: 1, n-9)$ & 3 & 1 & 3 & 1 & 3 & 1 & 3 & 2 & 3 & 1 & 4 & 2 & 3 & 2 & 3 & 1 & 3 & 3 \\
\hline Nervonic $(24: 1, n-9)$ & 223 & 25 & 228 & 24 & 226 & 24 & 218 & 30 & $242^{a, b}$ & 25 & $246^{a, b}$ & 20 & 217 & 24 & $254^{a, b}$ & 32 & $255^{a, b}$ & 37 \\
\hline \multicolumn{19}{|l|}{$n-6$ PUFA } \\
\hline LA $(18: 2, n-6)$ & 718 & 93 & 711 & 88 & 716 & 98 & 725 & 92 & $621^{a, b}$ & 99 & $561^{a, b, c}$ & 66 & 740 & 96 & $607^{a, b}$ & 69 & $550^{a, b, c}$ & 65 \\
\hline Eicosadinoic $†(20: 2, n-6)$ & 16 & 2 & 14 & 2 & 15 & 3 & 18 & 6 & 15 & 7 & 14 & 5 & 18 & 5 & 13 & 4 & 15 & 6 \\
\hline $\mathrm{DGLA}^{*}(20: 3, n-6)$ & 67 & 15 & 64 & 12 & 68 & 11 & 57 & 14 & 56 & 10 & 53 & 11 & 58 & 14 & 54 & 11 & 51 & 13 \\
\hline AA $(20: 4, n-6)$ & 665 & 59 & 644 & 72 & 645 & 47 & 667 & 97 & $599^{a, b}$ & 88 & $558^{a, b}$ & 55 & 672 & 74 & $535^{\mathrm{a}, \mathrm{b}, \mathrm{x}}$ & 45 & $498^{\mathrm{a}, \mathrm{b}, \mathrm{c}, \mathrm{x}}$ & 60 \\
\hline Adrenic $(22: 4, n-6)$ & 166 & 25 & 152 & 26 & 160 & 19 & 161 & 26 & $131^{a, b}$ & 17 & $132^{a, b}$ & 14 & 159 & 23 & $108^{\mathrm{a}, \mathrm{b}, \mathrm{x}}$ & 18 & $105^{\mathrm{a}, \mathrm{b}, \mathrm{x}}$ & 17 \\
\hline Osbond $(22: 5, n-6)$ & 29 & 8 & 25 & 5 & 28 & 5 & 29 & 6 & $20^{\mathrm{a}, \mathrm{b}}$ & 4 & $21^{a, b}$ & 5 & 31 & 6 & $17^{\mathrm{a}, \mathrm{b}, \mathrm{x}}$ & 4 & $16^{a, b, x}$ & 4 \\
\hline \multicolumn{19}{|l|}{$n-3$ PUFA } \\
\hline ALA $\ddagger(18: 3, n-3)$ & 8 & 3 & 8 & 2 & 8 & 3 & 9 & 2 & 10 & 2 & 9 & 2 & 9 & 2 & 10 & 2 & 9 & 3 \\
\hline $\operatorname{EPA}(20: 5, n-3)$ & 17 & 6 & 20 & 7 & 19 & 6 & 14 & 3 & $45^{\mathrm{a}, \mathrm{b}}$ & 9 & $78^{a, b, c}$ & 17 & 15 & 4 & $51^{a, b}$ & 11 & $91^{\mathrm{a}, \mathrm{b}, \mathrm{c}, \mathrm{x}}$ & 26 \\
\hline DPA $(22: 5, n-3)$ & 74 & 12 & 80 & 15 & 79 & 13 & $70^{\mathrm{a}}$ & 8 & $77^{\mathrm{b}}$ & 11 & $83^{\mathrm{b}}$ & 12 & $69^{a}$ & 10 & 73 & 10 & $86^{\mathrm{b}, \mathrm{c}}$ & 12 \\
\hline $\mathrm{DHA}(22: 6, n-3)$ & 134 & 20 & 140 & 31 & 136 & 28 & 143 & 23 & $207^{a, b}$ & 36 & $217^{a, b}$ & 33 & 158 & 25 & $228^{a, b}$ & 30 & $244^{a, b, x}$ & 45 \\
\hline
\end{tabular}

CON, control; EXP-Mod, experimental, moderate $n$-3 supplemented group; EXP-High, experimental, high $n$-3 supplemented group; LA, linoleic acid; DGLA, dihomo- - -linolenic acid; AA, arachidonic acid; ALA, $a$-linolenic acid,

Mean value was significantly different: ${ }^{\mathrm{a}} P<0.05$ from corresponding group average for week $0,{ }^{\mathrm{b}} P<0.05$ from average values for $\mathrm{CON}$ at the same time point, ${ }^{\mathrm{c}} P<0.05$ from average values for EXP-Mod at the same time point, ${ }^{\mathrm{x}} P<0.05$ from

* Main effect of time for palmitic, palmitoleic and DGLA, week $0>$ weeks 5 and 10.

† Main effect of group for eicosadinoic, behenic, lignoceratic and eicosadinoic acids, CON> EXP-Mod, EXP-High.

$¥$ Main effect of time for ALA, week $0<$ week 5 and week 10 . 
dietary intervention are shown in Table 4. Changes in erythrocyte fatty acid concentrations qualitatively mirrored the changes in plasma fatty acids during the intervention, with a few exceptions. Erythrocyte MUFA concentration increased (principally oleic, vaccenic and nervonic acids) in the EXP-Mod and EXP-High group by week 5 of the intervention and remained increased through week 10, in contrast to plasma concentrations which were unchanged. Also, the decrease in erythrocyte $n$ - 6 PUFA in both experimental groups during the intervention appeared somewhat more pronounced than the decrease in plasma values, in that $n-6$ PUFA concentrations continued to decrease between weeks 5 and 10, whereas there was no

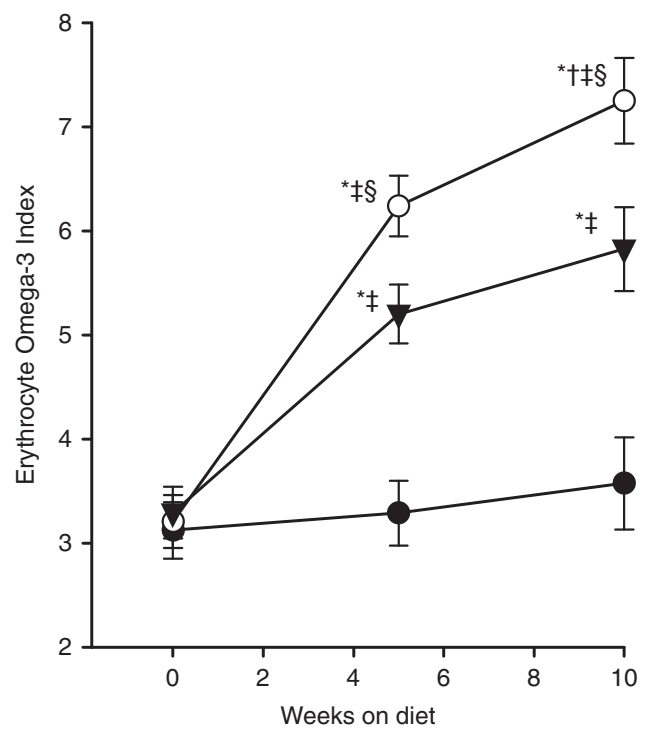

Fig. 2. Omega-3 Index of participants in control (CON, - - ) , experimental, moderate $n-3$ supplemented group (EXP-Mod, $\longrightarrow-$ ) and experimental-high $n$-3 supplemented group (EXP-High, - - ) before beginning the dietary intervention, and after 5 and 10 weeks on the intervention diets. * Significantly different from the corresponding group average for week $0 \quad(P<0.05)$ $\dagger$ Significantly different from the corresponding group average for week 5 $(P<0.05)$. $¥$ Significantly different from $\mathrm{CON}$ at the same time point $(P<0.05)$. $\S$ Significantly different from EXP-Mod at the same time point $(P<0.05)$. additional decrease in plasma concentrations after week 5 . Finally, the $n-3$ HUFA, DHA, was greater for the EXP-HIGH than EXP-Mod participants, at both weeks 5 and 10, whereas the corresponding differences in plasma DHA were not statistically significant. Fig. 2 depicts the Omega-3 Index calculated for each group before the intervention and after weeks 5 and 10. There were no differences in Omega-3 Index between groups before the intervention, and the Omega-3 Index of the CON group remained unchanged throughout the intervention. However, after 5 weeks of the intervention, Omega-3 Index of the EXP-Mod and EXP-High groups was higher than during week 0 , and Omega-3 Index in the EXP-High was higher than in EXP-Mod. Omega-3 Index of the EXP-High group continued to increase through week 10 of the intervention, whereas in the EXP-Mod group, the week 10 value was unchanged from the week 5 value.

\section{Blood lipids}

There were no significant differences between groups in measures of fasting plasma TAG, total cholesterol, LDL or HDL at any time, and none of the groups exhibited significant changes in any of the plasma lipid values during the intervention (data not shown). All values for these parameters were within normal clinical ranges for healthy young adults.

\section{Mood/emotional state}

Measures describing mood and emotional state are reported in Table 5. There were no differences between groups in any of the mood or emotional state measures at any time during the study. Although the vigour and fatigue scores from the POMS appeared to vary between weeks (significant main effect), there were no significant pairwise differences between means of any group over time, nor were there any significant interactions between group and week effects. No consistent relationship between plasma or erythrocyte fatty acids and measures of mood or emotional state was apparent, nor were any relationships between changes in plasma or erythrocyte HUFA with measures of mood or emotional status.

Table 5. Mood and emotional state assessments

(Mean values and standard deviations)

\begin{tabular}{|c|c|c|c|c|c|c|c|c|c|c|c|c|c|c|c|c|c|c|}
\hline & \multicolumn{6}{|c|}{ Week 0} & \multicolumn{6}{|c|}{ Week 5} & \multicolumn{6}{|c|}{ Week 10} \\
\hline & \multicolumn{2}{|c|}{$\mathrm{CON}$} & \multicolumn{2}{|c|}{ EXP-Mod } & \multicolumn{2}{|c|}{ EXP-High } & \multicolumn{2}{|c|}{ CON } & \multicolumn{2}{|c|}{ EXP-Mod } & \multicolumn{2}{|c|}{ EXP-High } & \multicolumn{2}{|c|}{$\mathrm{CON}$} & \multicolumn{2}{|c|}{ EXP-Mod } & \multicolumn{2}{|c|}{ EXP-High } \\
\hline & Mean & SD & Mean & SD & Mean & SD & Mean & SD & Mean & SD & Mean & SD & Mean & SD & Mean & SD & Mean & SD \\
\hline \multicolumn{19}{|l|}{ POMS } \\
\hline Tension & $1 \cdot 7$ & $2 \cdot 0$ & $1 \cdot 8$ & $2 \cdot 0$ & $1 \cdot 3$ & $2 \cdot 0$ & 1.4 & $1 \cdot 8$ & 1.4 & $1 \cdot 8$ & $1 \cdot 3$ & 1.7 & 0.9 & $1 \cdot 1$ & 1.4 & $2 \cdot 2$ & $1 \cdot 1$ & $2 \cdot 1$ \\
\hline Depression & 0.3 & 0.8 & 0.7 & $1 \cdot 2$ & 0.7 & $1 \cdot 1$ & 0.3 & 0.9 & 0.6 & $1 \cdot 1$ & $1 \cdot 1$ & $2 \cdot 1$ & 0.4 & $0 \cdot 8$ & 1.0 & 1.6 & 0.7 & 1.9 \\
\hline Anger & 0.6 & 1.0 & 1.2 & $2 \cdot 2$ & 0.8 & 1.4 & 1.0 & 1.4 & 1.6 & 4.0 & 1.4 & $2 \cdot 0$ & 0.5 & 1.0 & 1.0 & $1 \cdot 7$ & $1 \cdot 1$ & 1.8 \\
\hline Vigour* & 7.4 & 3.5 & 8.6 & 3.6 & $9 \cdot 1$ & 3.9 & $6 \cdot 2$ & 4.8 & 7.5 & 4.0 & 6.9 & 4.8 & $6 \cdot 8$ & $4 \cdot 7$ & 8.2 & 4.0 & $7 \cdot 0$ & $4 \cdot 1$ \\
\hline Fatigue* $^{\star}$ & 3.0 & 2.9 & 4.0 & 3.5 & 3.7 & 3.4 & $5 \cdot 0$ & 4.0 & 3.9 & 3.7 & 4.6 & 4.0 & 3.0 & $2 \cdot 8$ & 3.5 & 3.9 & 4.4 & $3 \cdot 1$ \\
\hline Confusion & $2 \cdot 8$ & 1.5 & $3 \cdot 2$ & $2 \cdot 4$ & 3.4 & 1.9 & $3 \cdot 2$ & $2 \cdot 1$ & $2 \cdot 9$ & 1.6 & $3 \cdot 3$ & $1 \cdot 7$ & $2 \cdot 8$ & 1.5 & 2.9 & $2 \cdot 3$ & $3 \cdot 0$ & $2 \cdot 4$ \\
\hline STAI-S & 29.5 & $7 \cdot 4$ & $29 \cdot 3$ & 8.5 & $29 \cdot 1$ & $7 \cdot 2$ & $29 \cdot 7$ & 8.7 & $29 \cdot 8$ & 8.5 & $30 \cdot 9$ & $7 \cdot 7$ & $28 \cdot 8$ & $7 \cdot 8$ & $30 \cdot 3$ & $10 \cdot 9$ & $29 \cdot 3$ & $9 \cdot 8$ \\
\hline STAI-T & $32 \cdot 6$ & 6.9 & 32.5 & $8 \cdot 2$ & $32 \cdot 3$ & 8.9 & 31.2 & $6 \cdot 8$ & $31 \cdot 8$ & 8.6 & $32 \cdot 2$ & $8 \cdot 2$ & $29 \cdot 3$ & 3.7 & $31 \cdot 8$ & 8.6 & $31 \cdot 7$ & 9.8 \\
\hline BDI & 3.3 & $4 \cdot 3$ & 3.5 & $4 \cdot 1$ & 4.0 & 4.4 & 4.4 & 4.9 & $2 \cdot 8$ & 3.9 & 4.6 & $5 \cdot 2$ & 3.4 & 3.7 & 3.0 & 4.5 & $5 \cdot 0$ & $6 \cdot 4$ \\
\hline
\end{tabular}

CON, control; EXP-Mod, experimental, moderate $n$-3 supplemented group; EXP-High, experimental, high $n$-3 supplemented group; POMS, Profile of Mood States; STAI-S, state anxiety; STAI-T, trait anxiety; BDI, Beck Depression Inventory.

* Main effect of week on vigour and fatigue, but no significant pairwise comparisons. 


\section{Discussion}

Previous studies ${ }^{(17-19)}$ demonstrate that dietary interventions which lower intake of $n$ - 6 PUFA can improve $n$ - 3 HUFA status in the body, with or without a concomitant increase in $n-3$ PUFA intake. However, those studies employed fairly restrictive regimens and supplementation to achieve major adjustments in dietary intake that are impractical for implementation in dining facilities serving people who are eating ad libitum. Our study is the first to evaluate the efficacy of a more practical dietary intervention for improving $n-3$ HUFA status that is suitable for implementation in collective group feeding settings like cafeterias and military garrison dining facilities. We investigated how swapping specific foods and food ingredients in the US Military's standard, 28-day Garrison Dining Facility Menu affected blood fatty acid and lipid profiles of participants consuming these foods. Overall, our findings indicate that this dietary intervention improved plasma and tissue $n-6$ and $n-3$ HUFA profiles in healthy, young adults.

In the experimental diets, the primary foods swapped included eggs, chicken and grass-fed beef from animals raised on feeds having high oleic acid content and low LA content, which replaced eggs, chicken and beef from animals raised on traditional (i.e. high LA content) used in the CON diets. In addition, the tuna fish used for the experimental diets was packed in olive oil whereas the standard tuna used in the CON diets was packed in soyabean oil. Also, the standard salad dressings, mayonnaise and peanut butter used in the CON diet were replaced with matching products made with high oleic soya oil and fortified with $n$ - 3 fatty acids, and wherever standard soyabean oil or margarine was used in CON diet recipes, high oleic soya oil and butter were substituted in experimental diet recipes. The control and experimental diets were indistinguishable to study participants and research staff (both blinded to the intervention assignment), and participants in all three groups rated their diet positively. As a result of the dietary intervention, participants in the two experimental groups consumed greater amounts of oleic acid and $n$ - 3 HUFA and less $n$-6 PUFA than consumed by the CON group (Table 2). In addition, the EXP-High group received a daily smoothie fortified with $n$-3 PUFA, so they consumed greater amounts of $n$-3 PUFA than the EXP-Mod and CON groups (Table 2), who received a same non-fortified, placebo smoothie.

Consistent with our hypothesis, both of the intervention diets improved $n$-3 HUFA status in our participants. Although plasma and erythrocyte concentrations of EPA and DHA in participants consuming the CON diet remained unchanged, plasma and erythrocyte concentrations of EPA and DHA and the Omega-3 Index increased in both experimental groups after 5 weeks on the intervention diet, and concentrations in both groups were higher than in the CON group. The additional dietary $n-3$ HUFA provided to the EXP-High group in the daily fortified smoothie caused erythrocyte and plasma EPA and DHA concentrations and the Omega-3 Index to increase even more than the increases exhibited by participants in the EXP-Mod group. Continuing the intervention diet through the 10 week had no additional effect on plasma and erythrocyte EPA and DHA concentrations in the EXP-Mod group, but erythrocyte EPA and
DHA concentrations and the Omega- 3 Index in the EXP-High group who received the daily $n-3$ HUFA fortified smoothie continued to increase between the 5 th and 10th week on the diet. Thus, the swapping approach we employed to decrease LA acid and increase $n-3$ HUFA in diets produced a substantial improvement in tissue $n$-3 HUFA status in the experimental groups in only 5 weeks.

Both experimental groups also exhibited reductions in plasma and erythrocyte LA and AA compared with before starting the diet (week 0), and compared with the CON group at week 5. Not all previous investigations have observed reductions in tissue AA with this type of intervention. Wood et al. ${ }^{(17)}$ reported that reducing dietary n-6 PUFA intake produced reductions in plasma and erythrocyte LA without any change in AA. However, the intervention diet in that study only lasted 4 weeks, which may have been insufficient to produce a reduction in AA. In addition, in the study reported by Wood et $a l .{ }^{(17)}$, LA intake of participants before beginning the intervention diet averaged $4.6 \%$ energy intake, and the 4-week intervention only reduced LA intake to $2.0 \%$ energy intake. In contrast, pre-study LA intakes of our participants averaged $7.5 \%$ energy intake (pre-study 24-h recall, data not shown). Further, during our 10-week study, daily LA intake of CON participants averaged $8.7 \%$ energy intake, compared with 3.3 and $3.2 \%$ for the EXP-Mod and EXP-High participants. Thus, the reduction in plasma and erythrocyte AA exhibited by the participants in the two experimental groups in our study may be at least partially attributable to the more pronounced reduction in LA intake compared with the intervention used by Wood et al. ${ }^{(17)}$. In addition, $n-3$ HUFA intake of participants in both of our experimental groups was increased compared with intakes in the CON group (see Table 2), and others have reported that decreased $n$ - 6 PUFA intake combined with increased $n$ - 3 HUFA intake produced a decline in plasma ${ }^{(18)}$ and erythrocytes ${ }^{(19)}$ AA levels, whereas diets that only reduced $n-6$ PUFA did not alter tissue AA levels ${ }^{(18,19)}$. Increasing dietary $n-3$ HUFA while dietary LA is also reduced is thought to increase competition for the desaturation and elongation enzymes in tissues that is required for metabolism of $n-3$ and $n-6$ fatty acids such that formation of DHA is favoured and formation of AA is reduced. Finally, besides having lower LA content, the foods in the intervention diets provided to the EXP-Mod and EXP-High participants in our study were lower in AA than foods in the CON diet. Therefore, our findings confirm and extend earlier findings by demonstrating that tissue (plasma and erythrocyte) AA levels can be reduced in only 5 weeks when both a reduction in dietary LA (and AA) and an increase in dietary $n$ - 3 HUFA are sustained. However, our data do not address whether a reduction in tissue AA levels would have clinical implications vis-à-vis inflammation as others have speculated ${ }^{(18,39,40)}$.

Some research indicates that risk of sudden cardiac death (SCD) and/or CHD may be lower when SFA intakes are reduced and MUFA and LA intakes are increased ${ }^{(41-43)}$. However, despite the substantial reduction in LA intake and increase in MUFA (primarily oleic acid) in the diet consumed by our experimental groups, we observed no changes in fasting plasma TAG, total cholesterol, LDL or HDL, biomarkers thought to reflect risk of SCD or CHD. On the other hand, erythrocyte Omega-3 Index 
increased to over $5 \%$ by the 5 th week on the diet in the EXP-Mod participants in our study, and Omega-3 Index increased even more in the EXP-High group who consumed the $n-3$ fortified smoothie, reaching over $7 \%$. Those changes correspond to $n-3$ HUFA levels associated with a 50-70\% reduction in risk of SCD among 278 participants in the Physicians Health Study who were followed for 17 years $^{(44)}$. Further, Harris \& von-Schacky ${ }^{(28)}$ examined the relationship between the tissue Omega-3 Index and $\mathrm{CHD}$ risk observed in a number of epidemiological and randomised controlled intervention studies, and concluded that levels $\geq 4 \%$ represented the threshold for protection, and levels $\geq 8 \%$ provided the greatest risk reduction. Presumably the changes in $n$ - 3 HUFA status we observed in our participants would have to be sustained much longer to achieve a meaningful reduction in SCD and $\mathrm{CHD}$ risk.

Dietary intake of $n-3$ and $n-6$ PUFA has also been suggested to modulate mood and psychological status ${ }^{(10-12,14,45)}$. In our study, we observed no differences in indices of mood and emotional state between the groups at any time, before or after consuming the intervention diets for 5 or 10 weeks. Further we observed no association between changes in tissue HUFA levels and mood during the study. However, the effects of anti-depressive treatments are related to the severity of depressive symptoms exhibited by the patient ${ }^{(46,47)}$, and our participants were all young, healthy adults, and scores for mood and emotional state assessments were at the very lowest end of the response scales, indicating that these participants had no underlying mood disorders or symptoms of anxiety or depression. Nevertheless, our observations indicate that the experimental diets had no adverse effects on mood or symptoms of anxiety or depression.

A unique aspect of this study was that we evaluated a very practical dietary intervention that would be feasible for implementation in group dining facilities which cater to a diverse group of diners with a wide range of food likes and dislikes. As described previously, we used a swapping process to substitute certain high oleic acid/low LA foods for the 'like' high LA acid containing foods typically used in eight of the menus from the US Military's standard, 28-day Garrison Dining Facility Menu. Almost all $(92.8 \%)$ of the reduction in the LA intake for the two experimental groups resulted from swapping high LA containing condiments and sauces in the standard menu with low LA/high oleic acid replacement items identical in appearance. Disregarding the $n-3$ fortified smoothie consumed by the EXP-High participant group, most of the increased $n$ - 3 HUFA intake for the experimental groups was achieved by swapping specially produced, high $n-3$ fatty acid containing eggs (33.3\%) and condiments and sauces $(36 \cdot 3 \%)$ for the ordinary eggs, condiments and sauces used in the standard menu consumed by the control participants. Swapping tuna fish packed in olive oil to replace the tuna fish packed in soyabean oil in the standard diet accounted for another $26.9 \%$ of the increase in $n-3$ fatty acid intake in the experimental diets. The specialty chicken and grass-fed beef products from animals raised on diets that aimed to lower $n-6$ and raised $n-3$ fatty acid content of the meat actually accounted for only a small part of the increased $n-3$ fatty acid intake ( 3.3 and $0.3 \%$, respectively) when they were used in the experimental diets to replace the ordinary beef and chicken in the standard diet. Thus, the experimental diets affected $n-3$ fatty acid status by both the direct effect of increased consumption of preformed $n-3$ HUFA, as well as to the secondary effect of decreased competition from LA for the enzymes that catalyse conversion of ALA into $n-3$ HUFA. This is apparent from the observation that even though erythrocyte levels of $n$-3 HUFA and erythrocyte Omega- 3 Index values rose higher in participants provided the smoothie fortified with $n-3$ HUFA, $n-3$ HUFA levels more than doubled and Omega-3 Index rose by about $60 \%$ in participants provided only the swapped diet without the fortified smoothie.

This study had some limitations. The participants were healthy young American adults, who typically consumed diets relatively high in $n-6$ fatty acids and low in $n-3$ fatty acids, so results may not be generalised to populations subsisting on a substantially different diet. Compliance with the dietary interventions imposed on our participants was high, since all meals consumed over the 10-week study were prepared by the study team, served in measured portions designed to maintain neutral energy balance for each individual, and most of the meals served each participant onsite were consumed in their entirety under the direct observation of study staff. Whether providing the intervention diets to consumers eating ad libitum would have the same quantitative impact on body $n$ - 3 HUFA status as was observed in our participants is not known. Finally, our findings do not address the clinical significance of the changes in $n-3$ HUFA status that the intervention diets produced for patients suffering depression or metabolic disorders (e.g. hyperlipidaemia), since our participants were all healthy, not suffering any illnesses that might be impacted by changes in $n-3$ fatty acid status, and the intervention only lasted for 10 weeks. Future studies should investigate whether different patient populations at higher risk of for $\mathrm{CVD}, \mathrm{SCD}$, psychiatric or neuroinflammatory disorders would exhibit more pronounced clinical effects with long duration dietary interventions.

\section{Conclusion}

In conclusion, we have demonstrated that swapping high LA containing foods and food ingredients typically used in the US Military's standard, 28-day Garrison Dining Facility Menu, and replacing them with similar high oleic acid/low LA and high $n-3$ fatty acid foods substantially improved $n-6 / n-3$ blood fatty acid status of people consuming these foods in a relatively short period of time. The specialty meat and eggs used in the experimental diets can be produced at a commercial scale, with only a modest increase in retail cost. The shelf life of these specialty foods is equal to current products. The specialty condiments, high oleic oils and $n-3$ fortified smoothie used in this study are already commercially available. The modest dietary adjustments are well accepted by diners, and are feasible for implementation in group feeding settings such as military dining facilities and other types of cafeterias, with little added cost.

\section{Acknowledgements}

The authors wish to thank the Pennington Biomedical Research Center staff for their dedicated support of this research project; specifically the contributions of Susan Mancuso, Renee Puyau, Courtney Brock, Grace Bella, Ray Allen and Frank Greenway. The authors also wish to thank the multiple companies who 
provided in-kind support, notably, Belovo Inc. (Christopher ${ }^{\mathrm{TM}}$ Eggs and Natures IQTM Chicken Meat), Omega Foods LLC (Various Dressings, Alfredo and Pasta Sauces, Mayonnaise and Peanut Butter), DuPont Pioneer (Plenish ${ }^{\mathrm{TM}}$ Cooking Oils), SmartFish AS (NutriFriend Smoothie Drinks), Vital Choice Wild Seafood \& Organics, Inc. (Albacore Tuna), Slanker's Grass-Fed Meats (Grass-Fed Beef) and Wenger's Feed Mills, Inc. (Poultry Feed Ingredients and Blending).

This study was supported by funding provided by the US Army Medical Research and Materiel Command and by the Intramural Program of the National Institute on Alcohol Abuse and Alcoholism. In addition, in-kind support was provided by Belovo Inc., Dupont Inc., Omega Foods LLC, SmartFish AS, Vital Choice Wild Seafood \& Organics, Inc., Slanker's Grass-Fed Meats and Wenger's Feed Mills, Inc., as detailed above in the 'Acknowledgements' section.

The authors responsibilities were as follows: A. J. Y., B. P. M., J. R. H., C. M. C., M. R. H., N. M. J., S. J. M. designed the research and analysed the data; C. M. C. and N. M. J. conducted the research; A. J. Y. and S. J. M. wrote the manuscript and had primary responsibility for the final content; all authors read, edited and approved the final manuscript.

$\mathrm{MRH}$ is the President of Belovo, Inc. and he was responsible for coordinating the vendor support and their in-kind contributions; the other authors have no potential COI to report.

\section{Supplementary material}

For supplementary material/s referred to in this article, please visit https://doi.org/10.1017/S0007114517001003

\section{References}

1. Blasbalg TL, Hibbeln JR, Ramsden CE, et al. (2011) Changes in consumption of omega- 3 and omega- 6 fatty acids in the United States during the 20th century. Am J Clin Nutr 93, 950-962.

2. Ailhaud G \& Guesnet P (2004) Fatty acid composition of fats is an early determinant of childhood obesity: a short review and an opinion. Obes Rev 5, 21-26.

3. Guyenet SJ \& Carlson SE (2015) Increase in adipose tissue linoleic acid of US adults in the last half century. Adv Nutr $\mathbf{6}$, 660-664.

4. Ramsden CE, Zamora D, Leelarthaepin B, et al. (2013) Use of dietary linoleic acid for secondary prevention of coronary heart disease and death: evaluation of recovered data from the Sydney Diet Heart Study and updated meta-analysis. BMJ 346, e8707.

5. Ramsden CE, Zamora D, Majchrzak-Hong S, et al. (2016) Re-evaluation of the traditional diet-heart hypothesis: analysis of recovered data from Minnesota Coronary Experiment (1968-73). BMJ 353, 17.

6. Harris WS, Kris-Etherton PM \& Harris KA (2008) Intakes of long-chain omega-3 fatty acid associated with reduced risk for death from coronary heart disease in healthy adults. Curr Atheroscler Rep 10, 503-509.

7. Lavie CJ, Milani RV, Mehra MR, et al. (2009) Omega-3 polyunsaturated fatty acids and cardiovascular diseases. J Am Coll Cardiol 54, 585-594.

8. Kiecolt-Glaser JK, Belury MA, Andridge R, et al. (2011) Omega-3 supplementation lowers inflammation and anxiety in medical students: a randomized controlled trial. Brain Behav Immun 25, 1725-1734.
9. Ferguson JF, Mulvey CK, Patel PN, et al. (2014) Omega-3 PUFA supplementation and the response to evoked endotoxemia in healthy volunteers. Mol Nutr Food Res 58, 601-613.

10. Hallahan B, Hibbeln JR, Davis JM, et al. (2007) Omega-3 fatty acid supplementation in patients with recurrent self-harm. Single-centre double-blind randomised controlled trial. $\mathrm{Br} \mathrm{J}$ Psychiatry 190, 118-122.

11. Hibbeln JR (1998) Fish consumption and major depression. Lancet 351, 1213.

12. McNamara RK, Able J, Jandacek R, et al. (2010) Docosahexaenoic acid supplementation increases prefrontal cortex activation during sustained attention in healthy boys: a placebo-controlled, dose-ranging, functional magnetic resonance imaging study. $\mathrm{Am} \mathrm{J}$ Clin Nutr 91, 1060-1067.

13. Alvheim AR, Malde MK, Osei-Hyiaman D, et al. (2012) Dietary linoleic acid elevates endogenous 2-AG and anandamide and induces obesity. Obesity (Silver Spring) 20 , 1984-1994.

14. Lewis MD, Hibbeln JR, Johnson JE, et al. (2011) Suicide deaths of active-duty US military and omega- 3 fatty-acid status: a case-control comparison. J Clin Psychiatry 72, 1585-1590.

15. Defense Science Board (2013) Study on Technology and Innovation Enablers for Superiority in 2030. Washington, DC: Defense Science Board.

16. Hibbeln JR \& Gow RV (2014) The potential for military diets to reduce depression, suicide, and impulsive aggression: a review of current evidence for omega- 3 and omega- 6 fatty acids. Mil Med 179, 117-128.

17. Wood KE, Lau A, Mantzioris E, et al. (2014) A low omega-6 polyunsaturated fatty acid ( $n-6$ PUFA) diet increases omega-3 $(n-3)$ long chain PUFA status in plasma phospholipids in humans. Prostaglandins Leukot Essent Fatty Acids 90, 133-138.

18. Taha AY, Cheon Y, Faurot KF, et al. (2014) Dietary omega-6 fatty acid lowering increases bioavailability of omega-3 polyunsaturated fatty acids in human plasma lipid pools. Prostaglandins Leukot Essent Fatty Acids 90, 151-157.

19. MacIntosh BA, Ramsden CE, Faurot KR, et al. (2013) Low- $n-6$ and low- $n-6$ plus high- $n-3$ diets for use in clinical research. Br J Nutr 110, 559-568.

20. Marriott BP, Yu K, Majchrzak-Hong S, et al. (2014) Understanding diet and modeling changes in the omega-3 and omega- 6 fatty acid composition of US garrison foods for active duty personnel. Mil Med 179, 168-175.

21. US Office of Management and Budget (1997) Revisions to the Standards for the Classification of Federal Data on Race and Ethnicity. Washington, DC: US Office of Management and Budget.

22. Joint Culinary Center of Excellence (2016) US Military 28 Day Garrison Dining Menu. http://www.quartermaster.army.mil/ jccoe/Operations_Directorate/CSPD/Final_Revision_CONOPS_ Menu_MARCH_2012.pdf (accessed September 2016).

23. Armed Forces Recipe Service (2003) Index of Recipes. Washington, DC: US Army.

24. Mifflin MD, St Jeor ST, Hill LA, et al. (1990) A new predictive equation for resting energy expenditure in healthy individuals. Am J Clin Nutr 51, 241-247.

25. Folch J, Lees M \& Stanley GHS (1957) A Simple method for the isolation and purification of total lipides from animal tissues. J Biol Chem 226, 497-509.

26. Morrison WR \& Smith LM (1964) Preparation of fatty acid methyl esters + dimethylacetals from lipids with boron fluoride-methanol. J Lipid Res 5, 600-60.

27. Lin YH, Salem N, Wells EM, et al. (2012) Automated highthroughput fatty acid analysis of umbilical cord serum and application to an epidemiological study. Lipids $\mathbf{4 7}, 527-539$. 
28. Harris WS \& Von-Schacky C (2004) The Omega-3 Index: a new risk factor for death from coronary heart disease? Prev Med 39, 212-220.

29. McNair DM, Lorr M \& Droppleman LF (1971) Manual: Profile of Mood States. San Diego, CA: Educational and Industrial Testing Service.

30. McNair DM, Lorr M \& Droppleman LF (1981) Profile of Mood States Questionnaire. San Diego, CA: Educational and Industrial Testing Service.

31. Spielberger CD \& Reheiser EC (2004) Measuring Anxiety, anger, depression and curiosity as emotional states and personality traits with the STAI, STAXI and STPI. In Comprehensive Handbook of Psychological Assessment (Volume 2): Personality Assessment, pp. 74-80 [MJ Hilsenroth and M Hevsen, editors]. Hoboken, NJ: Wiley.

32. Beck AT, Steer RA \& Brown GK (1996) Manual for the Beck Depression Inventory - II. San Antonio, TX: Psychological Corporation.

33. Baron-Cohen S, Wheelwright S, Hill J, et al. (2001) The 'Reading the Mind in the Eyes' test revised version: a study with normal adults, and adults with Asperger syndrome or highfunctioning autism. J Child Psychol Psychiatry 42, 241-251.

34. Sprengelmeyer R, Young AW, Calder AJ, et al. (1996) Loss of disgust - perception of faces and emotions in Huntington's disease. Brain 119, 1647-1665.

35. Ekman P \& Friesen WV (1976) Pictures of Facial Effect. Palo Alto, CA: Consulting Psychologists' Press.

36. Williams JM, Mathews A \& MacLeod C (1996) The emotional Stroop task and psychopathology. Psychol Bull 120, 3-24.

37. van Honk J, Tuiten A, de Haan E, et al. (2001) Attentional biases for angry faces: relationships to trait anger and anxiety. Cogn Emot 15, 279-297.
38. Dougherty DM, Marsh DM \& Mathias CW (2002) Immediate and delayed memory tasks: a computerized behavioral measure of memory, attention, and impulsivity. Behav Res Methods Instrum Comput 34, 391-398.

39. Calder PC (2015) Marine omega-3 fatty acids and inflammatory processes: effects, mechanisms and clinical relevance. Biochim Biophys Acta 1851, 469-484.

40. Janssen CI \& Kiliaan AJ (2014) Long-chain polyunsaturated fatty acids (LCPUFA) from genesis to senescence: the influence of LCPUFA on neural development, aging, and neurodegeneration. Prog Lipid Res 53, 1-17.

41. Farvid MS, Ding M, Pan A, et al. (2014) Dietary linoleic acid and risk of coronary heart disease: a systematic review and meta-analysis of prospective cohort studies. Circulation $\mathbf{1 3 0}$, $1568-1578$

42. Harris WS, Assaad B \& Poston WC (2006) Tissue omega-6/ omega-3 fatty acid ratio and risk for coronary artery disease. Am J Cardiol 98, 19i-26i.

43. Sacks FM \& Katan M (2002) Randomized clinical trials on the effects of dietary fat and carbohydrate on plasma lipoproteins and cardiovascular disease. Am J Med 113, 13-24.

44. Albert CM, Campos H, Stampfer MJ, et al. (2002) Blood levels of long-chain $n-3$ fatty acids and the risk of sudden death. $N$ Engl J Med 346, 1113-1118.

45. Parletta N, Milte CM \& Meyer BJ (2013) Nutritional modulation of cognitive function and mental health. $J$ Nutr Biochem $\mathbf{2 4}$, 725-743.

46. Fournier JC, DeRubeis RJ, Hollon SD, et al. (2010) Antidepressant drug effects and depression severity: a patient-level meta-analysis. JAMA 303, 47-53.

47. Hallahan B, Ryan T, Hibbeln JR, et al. (2016) Efficacy of omega-3 highly unsaturated fatty acids in the treatment of depression. Br J Psychiatry 209, 192-201. 